\title{
The role of ultrasound in the management of breast disease
}

\author{
Ian C Bennett and Magdalena A Biggar
}

\author{
Princess Alexandra Hospital, Woolloongabba, Queensland 4102, Australia. \\ Correspondence to author via ASUM. Email authors@asum.com.au
}

\begin{abstract}
Surgeon-performed ultrasound has also been increasingly utilised by breast surgeons in particular, who find ultrasound an invaluable tool in the office to assist in the management of breast patients, and also in the operating room where it can be used to directly guide excision of impalpable breast lesions without the use of hookwires ${ }^{2,3}$. In the future, the role of ultrasound will continue to grow, particularly with the advent of 3-D ultrasound, and in the evolving area of breast lesion ablation techniques using modalities such as radiofrequency ablation or laser therapy. Ultrasound is a very important tool in the assessment and management of breast disease and it has dramatically changed the way breast cancer patients are managed.
\end{abstract}

\section{Introduction}

Over the past 30 years, the increasing sophistication of ultrasound technology has led to a remarkable explosion in the utilisation of ultrasound as a medical imaging tool and, in particular, its role in breast imaging has advanced considerably. Whereas in the late 1980s the role of breast ultrasound was limited to discerning whether a lesion was solid or cystic, modern linear-array high frequency ultrasound probes can discern many of the features of a lesion in great detail including often detecting the presence of microcalcification ${ }^{1}$. While the current role of ultrasound as a screening modality is somewhat controversial, it has a very important function in breast diagnostic workup, including not only its capacity as an imaging modality in itself, but also as a means of undertaking various types of needle biopsy interventions and in the placement of localisation hookwires to help guide surgical excision of impalpable breast lesions. Surgeon-performed ultrasound has also been increasingly utilised by breast surgeons in particular, who find ultrasound an invaluable tool in the office to assist in the management of breast patients, and also in the operating room where it can be used to directly guide excision of impalpable breast lesions without the use of hookwires ${ }^{2,3}$. In the future, the role of ultrasound will continue to grow, particularly with the advent of 3-D ultrasound, and in the evolving area of breast lesion ablation techniques using modalities such as radiofrequency ablation or laser therapy ${ }^{4}$.

\section{Modern ultrasound equipment}

Breast ultrasound is best performed with high frequency linear array electronically focused transducers with accompanying computer enhanced imaging. The typical operating frequencies which give best breast imaging are in the range of $7.5-15 \mathrm{MHz}$ and these allow the delineation of subtle lesion characteristics to assist in the discrimination between benign and malignant lesions. This high frequency $\mathrm{B}$ mode (two dimensional) imaging does however have a limited range of penetration to a depth of approximately $4-6 \mathrm{~cm}$, and thus is not suitable for imaging other larger areas such as the abdomen. Many of the modern smaller portable ultrasound units come with high frequency transducers making them very appropriate for breast ultrasound work.

\section{Breast ultrasound screening}

While presently there is no clear role for breast ultrasound screening in the general population, there have been some reports of ultrasound screening being successful in high-risk women. The ACRIN 6666 trial found that the addition of a single screening ultrasound to mammographic screening in high risk women increased the diagnostic yield from 7.6 cancers per 1000 women screened for mammography only to 11.8 cancers per 1000 women screened for combined ultrasound and mammography screening 5 . Thus the supplemental yield was 4.2 per 1000 women screened. However, the downside was there was an increase in the false positive rate. In a recent review of a high risk family history clinic in Brisbane that utilises a multimodality screening approach including clinical breast examination, breast ultrasound and mammography, it was noted that approximately $26 \%$ of the cancers detected in this series were found by ultrasound screening alone ${ }^{6}$.

\section{Diagnostic ultrasound imaging}

Breast ultrasound has its greatest role in the diagnostic workup of symptomatic patients. Breast ultrasound examination is typically performed with the patient in the supine position with the ipsilateral arm raised and with a pillow placed under the shoulder. Ample acoustic gel needs to be applied between the transducer and the skin and the probe is usually moved in a systematic radial fashion toward the nipple with any lesions being identified documented as a position on the clock face and designated as a number of centimetres away from the nipple. It is also important that the correct scan plane is recorded for the image and the size of the lesion is accurately demonstrated.

There are nine important ultrasound diagnostic criteria to distinguish between benign and malignant breast lesions: Shape: most benign lesions are either round or oval or they may be slightly lobulated, malignant lesions, however, tend to be quite irregular.

Orientation: a benign lesion may be round therefore having no axis at orientation (depth/width $(\mathrm{D} / \mathrm{W})$ ratio $=1)$. This is classical of a cyst. An oval benign lesion however classically lies with its longitudinal axis parallel to the skin (D/W ratio $<1$ ) and this is typical of a fibroadenoma. However a cancer 
Table 1: Ultrasound characteristics of common benign and malignant lesions.

\begin{tabular}{|l|l|l|l|}
\hline & Benign: Cyst & Benign: Fibroadenoma & Malignant: Carcinoma \\
\hline Shape & Round / Oval & Round / Oval & Irregular \\
\hline Orientation & Horizontal & Horizontal & Vertical \\
\hline Margins & Circumscribed & Circumscribed & Irregular, indistinct \\
\hline Echogenicity & Anechoic & Hypoechoic & Hypoechoic \\
\hline Internal echopatern & None & Homogeneous & Heterogeneous \\
\hline Compression & Shape change & $\begin{array}{l}\text { Internal echoes more } \\
\text { homogeneous }\end{array}$ & No change \\
\hline Retrotumour acoustics & Posterior enhancement & $\begin{array}{l}\text { No or slight posterior } \\
\text { enhancement; } \\
\text { Bilateral edge shadowing }\end{array}$ & Irregular posterior shadowing \\
\hline Vascularity & & None to low & Increased \\
\hline Surrounding architecture & Normal & Normal & Distorted \\
\hline
\end{tabular}

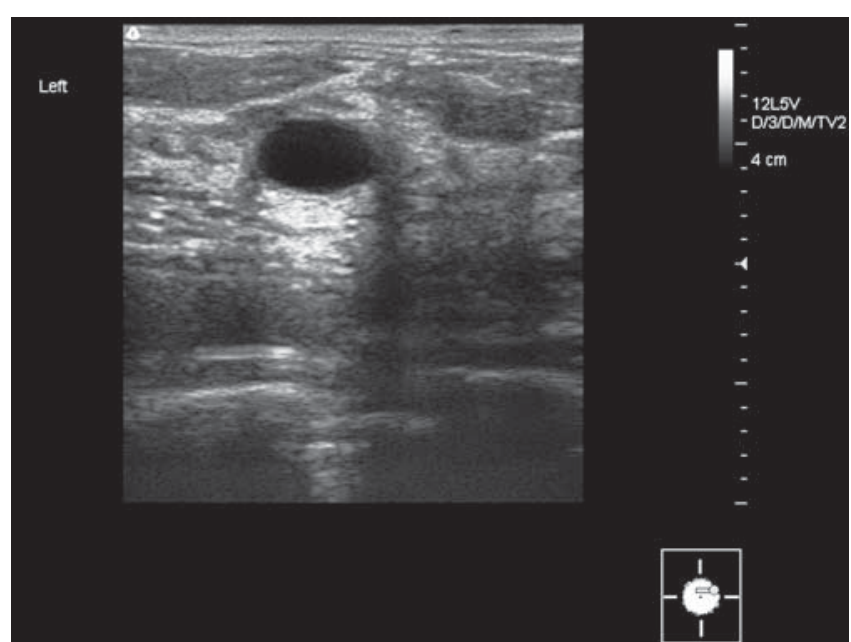

Fig. 1: Ultrasound image of a breast cyst - demonstrating bilateral edge shadowing and posterior acoustic enhancement.

often is taller than it is wide having its vertical axis at $90^{\circ}$ to the skin $(\mathrm{D} / \mathrm{W}$ ratio $>1)$.

Margins: benign lesions tend to have smooth margins whereas malignancies tend to be indistinct and jagged.

Echogenicity: a typical cyst is anechoic, a lipoma is hyperechoic and a solid lesion in hypoechoic, malignancies tend to be irregular hypoechoic lesions.

Internal echo pattern: a benign hypoechoic lesion will have a homogeneous internal echo pattern whereas a malignancy tends to be quite heterogeneous in its internal echo structure.

Compressibility: benign lesions tend to be compressible and change shape when downward pressure is applied with the transducer whereas malignant lesions tend to be unaltered by this process.

Retrotumour acoustic features: a cyst typically demonstrates posterior enhancement i.e. bright shadows on its deeper aspect, whereas a malignancy which is quite dense and which absorbs the soundwaves tends to demonstrate posterior shadowing.

Vascularity: malignancies tend to be hypervascular compared to benign lesions or surrounding parenchyma.

Interruption of surrounding architecture: malignancies typically interrupt and distort adjacent tissue planes, which is most evident with cicatrising lesions.

Table 1 summarises the typical ultrasound characteristics

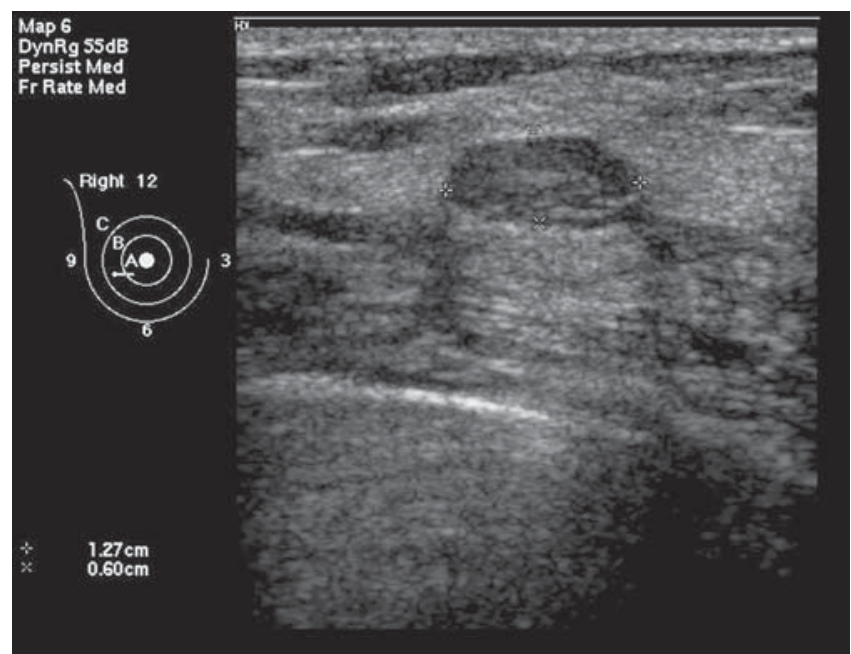

Fig. 2: Sonographic image of a fibroadenoma showing well defined margins and regular internal echoes.

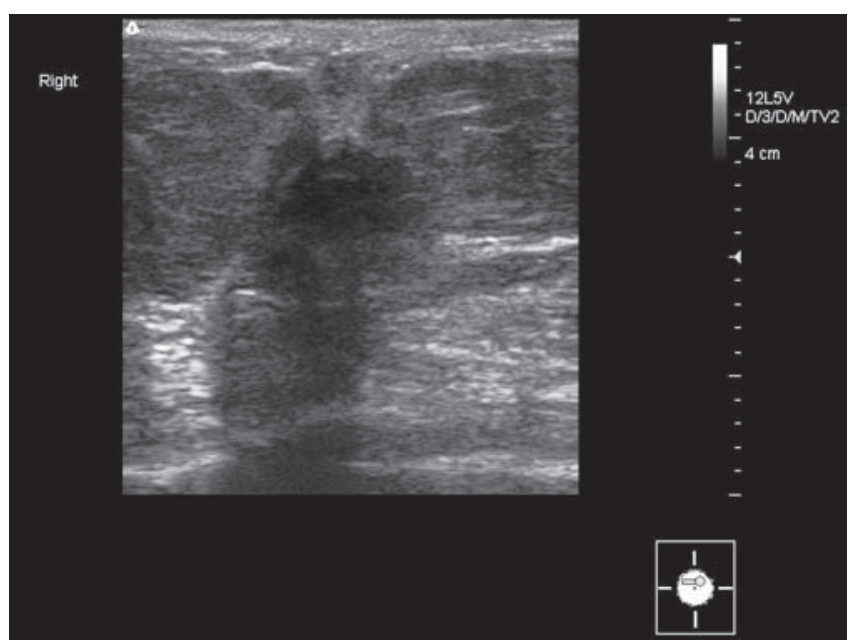

Fig. 3: Sonographic image of a malignant breast mass - an irregular hypoechoic lesion with posterior shadowing and distortion of surrounding parenchyma.

of a cyst, a benign fibroadenoma and a carcinoma. Typical examples of sonographic images of these lesions are depicted in Figs. 1-3.

\section{Diagnostic needle interventions}

Fine needle aspiration biopsies (FNAB) can be readily performed under ultrasound guidance as an outpatient 


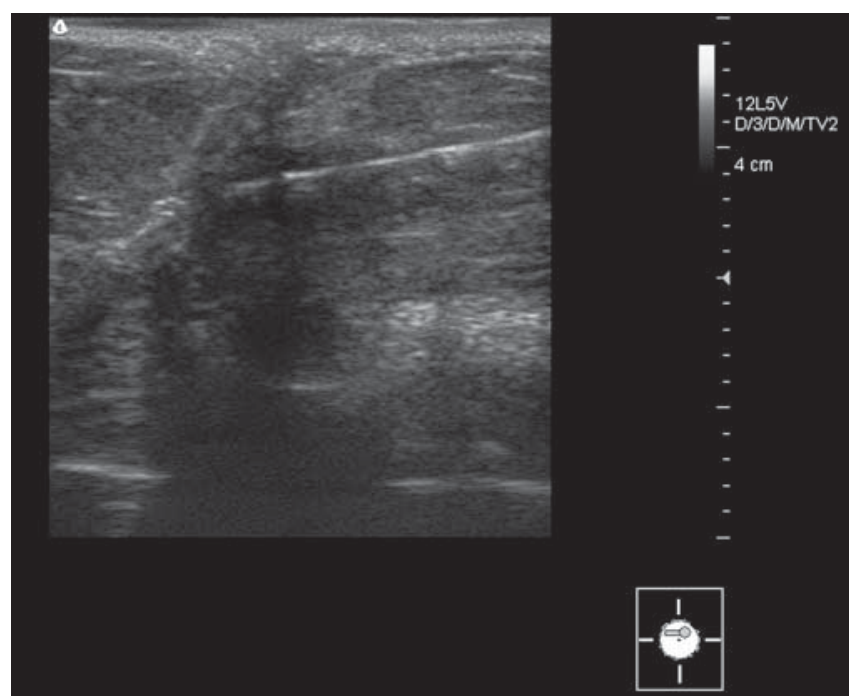

Fig. 4: Ultrasound-guided FNAB of malignant breast lesion.

procedure (Fig. 4). Fine needle aspiration is often performed with a 22 gauge needle and it is a useful technique for determining the nature of indeterminant hypoechoic lesions many of which will prove to be atypical cysts containing echogenic debris. Cyst aspiration under ultrasound control can confirm that the cyst has been completely extinguished.

Ultrasound-guided core needle biopsy entails the use of spring-loaded automated needles with an excursion throw which allows small cylinders of tissue to be cut and collected within the notch of the needle. Core biopsy has the advantage of obtaining a tissue sample for histological diagnosis. It is slightly more time consuming to perform than FNAB but can still be readily performed on an outpatient basis. After acquisition of each core sample the needle has to be removed from the breast to obtain the specimen and be re-inserted for further samples ${ }^{7}$. The biopsy guns typically used for ultrasound core needle biopsy range from 12 up to 14 gauge with a throw of $10-22 \mathrm{~mm}$. The needle is inserted under ultrasound guidance while holding the transducer in one hand and the biopsy gun in the other. This allows visualisation of the needle tip at the edge of the lesion and when the needle is deployed it is important to obtain documentation of the needle traversing the lesion. An adequate sample is usually taken with approximately 5-6 cores.

Vacuum assisted core biopsies (VAB) performed under ultrasound are able to extract more tissue as the vacuum which is created within the device is used to pull tissue into the sampling chamber where it is removed with a high speed internal rotating blade. The specimen is then usually suctioned to a chamber outside the breast where it can be retrieved. In this way multiple samples can be removed through a single insertion of the needle and this can often therefore enable complete removal of a benign imaged abnormality ${ }^{7,8}$. The vacuum assisted core biopsy needles are usually larger, typically 8 to 14 gauge, and there are now quite a number of these devices on the market, such as Mammotome ${ }^{\mathrm{TM}}$ (Ethicon Endo-surgery, Inc. CN, Ohiao, USA), Vacora ${ }^{\mathrm{TM}}$ (Bard Biopsy Systems, Tempe, AZ, USA), Atec ${ }^{\mathrm{TM}}$ (Hologic, Inc. Bedford, MA, USA), Eviva ${ }^{\mathrm{TM}}$ (Hologic, Inc Bedford, MA, USA) and EnCor ${ }^{\mathrm{TM}}$ (SenoRx, Inc. Irvine, CA, USA).

In the diagnostic workup and management of breast patients ultrasound also has an important role in the arena of hookwire localisation. Impalpable breast lesions which need to be surgically excised for diagnostic or therapeutic reasons would usually have a hookwire placed to the lesion under ultrasound guidance pre-operatively, so that in theatre the surgeon can be directed to the correct area. Confirmation of excision of the lesion with specimen sonography is a valid and appropriate technique ${ }^{9}$.

The management of breast abscesses by repeated ultrasound guided needle aspirations is also a relatively new methodology which has been increasingly utilised in recent years and which in many cases can avoid the need for surgical drainage ${ }^{10}$.

\section{Surgeon performed ultrasound}

Due to the availability of portable sophisticated ultrasound units many breast surgeons have embraced the new ultrasound technology and incorporated this into their dayto-day management of breast patients, in both the areas of office ultrasound and intra-operative ultrasound ${ }^{2,4}$.

Office ultrasound provides a significant convenience to the patient particularly as it allows immediate one-shop assessment of any problematic areas of the breast, which may have been detected either clinically or on recent radiology. Most surgeons in these circumstances will perform a targeted ultrasound of the area in question and, particularly for vague palpable abnormalities, this can often provide immediate reassurance to the patient with good clinical and sonographic correlation. The ultrasound experienced breast surgeon can also readily perform needle biopsies of any specific atypical areas. It must be stressed however that training and accreditation in surgeon-performed ultrasound is important as part of this process to ensure that patients are offered high quality care ${ }^{11}$.

The use of ultrasound intra-operatively also represents a new frontier of surgical intervention and involves the breast surgeon utilising ultrasound to localise and direct excision of an impalpable breast lesion, thus avoiding the need for preoperative hookwire placement. This technique is an alternative to the hookwire guided excision technique described above, and thus represents a major convenience for the patient. It also avoids the potential complications that can occur with hookwire localisations such as retained wire fragments and pnuemothorax. A recent review of intraoperative ultrasound guided breast excisions demonstrated the technique to be safe and effective for both benign and malignant breast lesions, with improved margins of excision being achieved in instances of resection of malignancies ${ }^{12}$.

Another developing area in the use of intra-operative of ultrasound is assistance with the localisation of axillary sentinel nodes in the technique of sentinel node biopsy for early breast cancers. Techniques currently used to localise the sentinel node include radio-isotope and patent blue dye, but the additional use of ultrasound placed into the axillary wound may also assist in the detection of the sentinel node. In the future this technique may be assisted with the development of contrast materials that are enhanced and visible on ultrasound ${ }^{13}$.

\section{Future directions}

New technical innovations of the future may include computer enhanced diagnosis which uses computer analysis to aid in the interpretation of images with the aim of 
decreasing inter-observer variability ${ }^{14,15}$. 3-D ultrasound imaging is also rapidly evolving and may assist in improving the accuracy of image directed biopsies. It may also assist with tumour volume estimations, a feature which could prove useful when monitoring tumour response to neoadjuvant chemotherapy ${ }^{16}$. The future management of early breast cancers may also see the development of ultrasound guided ablative techniques such as radio-frequency ablation or laser guided therapies which may, in selected cases, represent valid alternatives to surgery, with ultrasound playing a very critical role in the development of these new techniques ${ }^{2,3,17}$.

\section{Conclusion}

Ultrasound is a very important tool in the assessment and management of breast disease and it has dramatically changed the way breast cancer patients are managed. The application of ultrasound-guided procedures allows more precise evaluation of breast abnormalities and the use of these techniques continues to evolve in both investigative and therapeutic roles.

\section{References}

1 Stavros AT, Thickman D, Rapp CL, Dennis MA, Parker SH, Sisney GA. Solid breast nodules: use of sonography to distinguish between benign and malignant lesions. Radiology 1995; 196: 123-34.

2 Rubio IT, Henry-Tillman R, Klimberg VS. Surgical use of breast ultrasound. Surg Clin N Am 2003; 83: 771-88.

3 Thompson M, Klimberg VS. Use of ultrasound in breast surgery. Surg Clin N Am 2007; 87: 469-84.

4 Fine RE, Staren, ED. Updates in breast ultrasound. Surg Clin N Am 2004; 84: 1001-34.

5 Berg WA, Blume JD, Cormack JB, Mendelson EB, Lehrer D, BohmVelez M, et al. [ACRIN 6666] Combined screening with ultrasound and mammography vs mammography alone in women at elevated risk of breast cancer. JAMA 2008; 299 (18): 2151-63.
6 Bennett IC, Muller J, Cockburn L, Joshua H, Thorley G, Baker C, et al. Outcomes of multimodality breast screening for women at increased risk of familial breast cancer. World J Surg 2010; 34: 979-86.

7 O'Flynn EA, Wilson AR, Michael MJ. Image-guided breast biopsy: state-of-the art. Clin Radiol 2010; 65: 259-70.

8 Fine RE, Whitworth PW, Kim JA, Harness JK, Boyd BA, Burak WE. Low risk palpable breast masses removed using a vacuum-assisted hand-held device. Am J Surg 2003; 186: 362-7.

9 Vujovic P, Gianduzzo T, Archibald C, Bennett I. Ultrasonic specimen radiography for non-palpable breast lesions. Breast 2002; 11: 144-50.

10 Ulitzsch D. Nyman MK. Carlson RA. Breast abscess in lactating women: US-guided treatment. Radiology 2004; 232: 904-9.

11 Australian Society for Ultrasound in Medicine website: http://www. asum.au/site/index.php Qualifications; CCPU Certificate in Clinician Performed Ultrasound, 2010.

12 Bennett IC, Greenslade J, Chiam H. Intraoperative ultrasound-guided excision of nonpalpable breast lesions. World J Surg 2005; 29: 369_ 74.

13 Balleyguier C, Opolon P, Mathieu MC, Athanasiou A, Garbay JR, Delaloge S, Dromain C. New potential and applications of contrastenhanced ultrasound of the breast: own investigations and review of the literature. Eur J Radiol 2009; 69: 14-23.

14 Horsch K, Giger ML, Vyborny CJ, Benta LA. Performance of computer-aided diagnosis in the interpretation of lesions on breast sonography. Acad Radiol 2004; 11: 272-80.

15 Kuo WJ, Chang RF, Moon WK. Computer aided diagnosis of breast tumors with different US systems. Acad Radiol 2002; 9: 793-9.

16 Athanasiou A, Tardivon A, ollivier L, Thibault F, Khoury CE, Neuenschwander S. How to optimize breast ultrasound. Eur J Radiol 2009; 69: 6-13.

17 Schmitz AC, Gianfelice D, Daniel BL, Mali WP, van den Bosch MA. Image-guided focused ultrasound ablation of breast cancer: current status, challenges, and future directions. Eur Radiol 2008; 18: 1431-41. 